\title{
Gravitational Imprints of Flavor Hierarchies
}

\author{
Admir Greljo®, ${ }^{1, *}$ Toby Opferkuch $\oplus^{1, \dagger}$ and Ben A. Stefanek $\oplus^{2, \#}$ \\ ${ }^{1}$ Theoretical Physics Department, CERN, 1211 Geneva, Switzerland \\ ${ }^{2}$ PRISMA Cluster of Excellence and Mainz Institute for Theoretical Physics, \\ Johannes Gutenberg-Universität Mainz, 55099 Mainz, Germany
}

(Received 14 October 2019; accepted 16 April 2020; published 1 May 2020)

\begin{abstract}
The mass hierarchy among the three generations of quarks and charged leptons is one of the greatest mysteries in particle physics. In various flavor models, the origin of this phenomenon is attributed to a series of hierarchical spontaneous symmetry breakings, most of which are beyond the reach of particle colliders. We point out that the observation of a multipeaked stochastic gravitational wave signal from a series of cosmological phase transitions could well be a unique probe of the mechanism behind flavor hierarchies. To illustrate this point, we show how near future ground- and space-based gravitational wave observatories could detect up to three peaks in the recently proposed $P S^{3}$ model.
\end{abstract}

DOI: 10.1103/PhysRevLett.124.171802

Introduction.-The first direct detection of gravitational waves (GW) [1] was a stunning confirmation of the theory of general relativity and marked the discovery of the only messenger via which the universe can be probed back to the Planck era. To take advantage of this unique window into the universe, the next few decades will see a plethora of ground- and space-based gravitational wave observatories being built across twelve decades in frequency [2-9]. In addition to what can be learned on the astrophysical front, this experimental effort offers an immense opportunity to probe fundamental physics in the early universe. Indeed, many particle physics processes that produce a stochastic gravitational wave background have already been identified, such as the primordial spectrum expected from inflation [10-12], violent first order phase transitions (FOPTs) [13-38], cosmic strings [39-42], nonperturbative particle production [43-49], primordial black holes [50-52], etc. Many of these processes are expected to produce a GW spectrum with a single peak, with the notable exception being the nearly scale-invariant spectrum from inflation.

Not as frequently discussed is the possibility of observing a multipeaked gravitational wave signal, in either single or multiple experiments, and what such a signal might tell us about open puzzles in fundamental physics. One intriguing possibility is that a multipeaked signal could come from a series of sequential FOPTs. As the peak frequency of the GW spectrum from a first order phase

Published by the American Physical Society under the terms of the Creative Commons Attribution 4.0 International license. Further distribution of this work must maintain attribution to the author(s) and the published article's title, journal citation, and DOI. Funded by SCOAP ${ }^{3}$. transition is set by the vacuum expectation value (VEV) in the broken phase, the observation of a multipeaked signal could contain information about the scales of multiple spontaneous symmetry breakings (SSBs), with the first breaking giving the highest frequency peak and the last the lowest.

A longstanding question within fundamental physics is that of the flavor puzzle, which refers to why the standard model (SM) fermion Yukawa couplings are spread over so many orders of magnitude, with a top quark Yukawa that is $\mathcal{O}(1)$ but an electron Yukawa which is five orders of magnitude smaller. Just the quark sector alone has a hierarchy which covers four to five decades and also contains the puzzle of why the CKM mixing matrix is close to identity.

It has been proposed that the flavor hierarchies could be generated via a series of hierarchical SSBs [53-63]. These types of models typically associate flavor with a fundamental gauge symmetry at high energies. The SM fermion masses and mixings are then generated via spontaneous breaking of this gauge symmetry, usually in several steps. The aforementioned models are compatible with the lowest SSB occurring at the TeV scale, which is highly motivated as it is the scale currently being probed at colliders (perhaps also in order to explain flavor anomalies [64-72]). Interestingly enough, if this breaking occurs via a strongly FOPT, the resulting GW signal is in the sensitivity range of upcoming space-based interferometers such as LISA. Moreover, the higher breakings associated with light family mass generation may produce $\mathrm{GW}$ in the range of future ground-based interferometers such as Einstein telescope (ET) and cosmic explorer (CE). Such a scenario would lead to a spectacular signature involving a multipeaked $\mathrm{GW}$ signal, the peak frequencies of which contain information about the flavor hierarchies, spread across future GW 


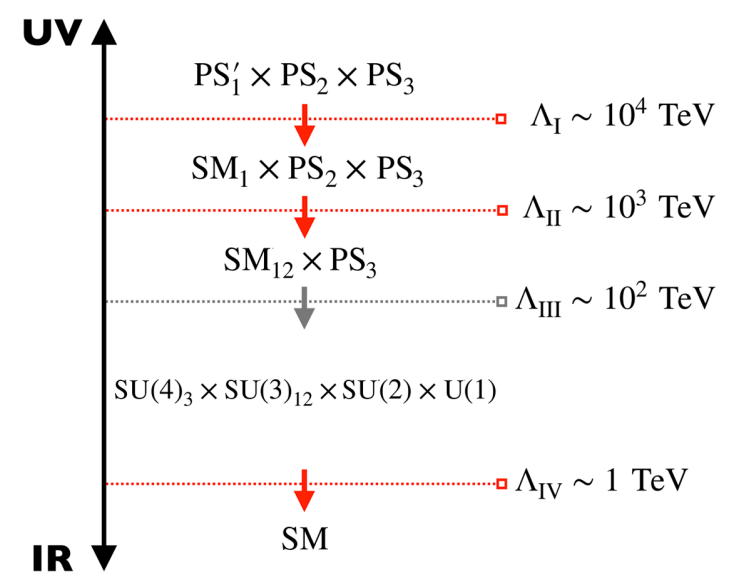

FIG. 1. Schematic view of the $\mathrm{PS}^{3}$ model detailed in the second section. Phase transitions marked with red arrows correspond to SU(4) breakings (see third section).

experiments covering four decades of frequency space. This separation in frequency can be roughly seen by taking the geometric mean of the quark masses of each family,

$$
\begin{array}{ccccc}
\sqrt{m_{t} m_{b}} & : & \sqrt{m_{s} m_{c}}, & : & \sqrt{m_{u} m_{d}} \\
1 & : & 10^{-2}, & : & 10^{-4}, \\
f_{\mathrm{LISA}}^{-1} & : & \ldots, & : & f_{\mathrm{ET}}^{-1} .
\end{array}
$$

To further develop this idea, we will take the PS $^{3}$ model of Ref. [63] as a concrete example in what follows, though the concept generalizes to many models which solve the flavor puzzle through a series of hierarchical SSBs.

Model example: Pati-Salam cubed.-As a prototypical example, we focus on the $\mathrm{PS}^{3}$ model first introduced in Ref. [63], see also [73]. Here, the original Pati-Salam gauge group [80] in higher-dimensional spacetime is deconstructed [81] onto 3 four-dimensional sites $\mathrm{PS}^{3} \equiv$ $\mathrm{PS}_{1} \times \mathrm{PS}_{2} \times \mathrm{PS}_{3}$, where each copy acts on one family of SM fermions. In particular, an entire SM family (including the right-handed neutrino) fits into two left- and right-chiral multiplets, $\Psi_{L}^{(i)} \equiv(\mathbf{4}, \mathbf{2}, \mathbf{1})_{i}$ and $\Psi_{R}^{(i)} \equiv(\mathbf{4}, \mathbf{1}, \mathbf{2})_{i}$, which embed quark and lepton doublets $Q_{L}^{(i)}$ and $L_{L}^{(i)}$ and singlets $u_{R}^{(i)}, \nu_{R}^{(i)}, d_{R}^{(i)}$, and $e_{R}^{(i)}$, respectively. The label $i=1,2,3$ denotes the corresponding gauge group $\mathrm{PS}_{i} \equiv[\mathrm{SU}(4) \times$ $\left.\mathrm{SU}(2)_{L} \times \mathrm{SU}(2)_{R}\right]_{i}$.

The model undergoes a series of SSBs occurring at different energy scales as illustrated in Fig. 1. The first breaking after inflation is triggered by the VEV of $\Sigma_{1}$ in 4 of SU(4) [82]. The subsequent breakings to the diagonal subgroups of neighboring sites are achieved by the appropriate scalar link fields in bifundamental representations, $\Phi_{i j}^{L, R}$ and $\Omega_{i j}$. More specifically, $\Phi_{i j} \mathrm{~s}$ are in $\mathbf{2}$ of the corresponding $\mathrm{SU}(2)_{i}$ and $\overline{\mathbf{2}}$ of $\mathrm{SU}(2)_{j}$, while similarly, $\Omega_{i j}$ is $(\mathbf{4}, \mathbf{2}, \mathbf{1})_{i} \times(\overline{\mathbf{4}}, \overline{\mathbf{2}}, \mathbf{1})_{j}$. Finally, the Higgs fields live at the third site, e.g., $H_{3} \equiv(\mathbf{1}, \mathbf{2}, \overline{\mathbf{2}})_{3}$.
Below the scale $\Lambda_{\mathrm{II}}$, the unbroken phase of the theory, $\mathrm{SM}_{12} \times \mathrm{PS}_{3}$, leads to an approximate $\mathrm{U}(2)$ flavor symmetry observed in the SM at low-energies [84]. The lower bound on this scale, $\Lambda_{\mathrm{II}} \gtrsim 10^{3} \mathrm{TeV}$, follows from stringent limits on flavor changing neutral currents (FCNC) induced by the heavy gauge bosons coupling to the first two generations [85-89]. At this level, Yukawa interactions are only allowed for the third family, $\mathcal{L} \supset \bar{\Psi}_{L}^{(3)} H_{3} \Psi_{R}^{(3)}$, predicting vanishing light-fermion masses and a CKM matrix equal to identity. The smallness of neutrino masses is achieved by the inverse seesaw mechanism [74]. The perturbation to this picture is obtained by higher-dimensional operators such as

$$
\begin{aligned}
& \mathcal{L}_{23}=\frac{1}{\Lambda_{\text {III }}} \bar{\Psi}_{L}^{(2)} \Omega_{23} H_{3} \Psi_{R}^{(3)}+\text { H.c., } \\
& \mathcal{L}_{12}=\frac{1}{\Lambda_{\mathrm{II}}^{2}} \bar{\Psi}_{L}^{(k)} \Phi_{k 3}^{L} H_{3} \Phi_{3 l}^{R} \Psi_{R}^{(l)}+\text { H.c. },
\end{aligned}
$$

after the link fields acquire VEVs. The leading $U(2)$ breaking spurion, following from the first term, generates the mixing of the third and light families, $\left|V_{t s}\right| \sim$ $\left\langle\Omega_{23}\right\rangle / \Lambda_{\text {III }}$, where $\left\langle\Omega_{23}\right\rangle \sim \Lambda_{\text {IV }}$. The light fermion masses are instead due to the second term, with the largest being $y_{c} \sim\left\langle\Phi_{23}^{L}\right\rangle\left\langle\Phi_{32}^{R}\right\rangle / \Lambda_{\mathrm{II}}^{2}$. Similarly, $y_{u}$ follows from $\Lambda_{\mathrm{I}}$, etc. The UV completion of the effective operators in Eq. (1) has been discussed in Refs. [63,74]. We assume the scales generating the operators to coincide with the preceding symmetry breaking scales, e.g., $\Lambda_{\mathrm{III}} \sim\left\langle\Phi_{23}\right\rangle$ and $\Lambda_{\mathrm{II}} \sim$ $\left\langle\Phi_{12}\right\rangle$. From here it follows that the four-step breaking, (i) $10^{4} \mathrm{TeV}$, (ii) $10^{3} \mathrm{TeV}$, (iii) $10^{2} \mathrm{TeV}$, and (iv) $1 \mathrm{TeV}$, is well compatible with the observed pattern of fermion masses and mixings at low energies [90]. As we will show later, the three SU(4) phase transitions naturally induce a stochastic GW signature within the reach of nextgeneration interferometers [91].

While we work in the deconstructed four-dimensional picture, the higher-dimensional model relates the hierarchy of quark and charged lepton masses to the stabilization mechanism of branes in the bulk [92]. Additionally, the higher-dimensional gauge symmetry justifies small scalar quartic couplings [93] leading to an almost classically scale invariant potential which is crucial to ensure strongly FOPTs as shown later.

Gravitational wave calculation.-Effective potential: To describe the first SSB in $\mathrm{PS}^{3}$ at the scale $\Lambda_{\mathrm{I}}$, we calculate in a simplified $4 \rightarrow 3$ model where SU(4) is broken to SU(3) by the VEV of a complex scalar $\Sigma$ in the fundamental representation of SU(4). The matter content includes one set of doublets $\Psi_{L}$ and $\Psi_{R}$, also in the fundamental representation of $\mathrm{SU}(4)$. In the $\mathrm{PS}^{3}$ model, scalar fields which break SU(4)s have suppressed Yukawa interactions and scalar crossquartics [94]. As a result, the relevant part of Lagrangian for the GW calculation depends only on a few parameters. More explicitly, 
$\mathcal{L}=\bar{\Psi} i \not D \Psi-\frac{1}{4}\left(F_{\mu \nu}^{a}\right)^{2}+\left|D_{\mu} \Sigma\right|^{2}+\lambda v^{2}|\Sigma|^{2}-\lambda|\Sigma|^{4}$,

with $D_{\mu}=\partial_{\mu}-i g A_{\mu}^{a} T^{a}$. Thus, the relevant parameters of the model are $g, \lambda$, and $v$. The breaking $\mathrm{SU}(4) \rightarrow \mathrm{SU}(3)$ occurs when the complex scalar $\Sigma$ acquires a VEV of the form $\langle\Sigma\rangle=(0,0,0, v / \sqrt{2})^{T}$. The seven broken generators correspond to a massive vector leptoquark $U_{\mu}$ and $Z^{\prime}$ gauge boson. The decomposition of $\Sigma$ under the unbroken SU(3) is $\mathbf{4}=\mathbf{3}+\mathbf{1}$, with the entire complex $\mathbf{3}$ and the imaginary part of $\mathbf{1}$ containing the leptoquark and $Z^{\prime}$ goldstones, respectively. The remaining degree of freedom $\operatorname{Re} \Sigma_{4} \equiv$ $\phi / \sqrt{2}$ is a massive radial mode. The full finite-temperature effective potential for $\phi$ is

$$
V_{\text {eff }}(g, \lambda, v, \phi, T)=V_{0}+V_{\mathrm{CW}}+V_{T \neq 0},
$$

where tree level potential $V_{0}$ is

$$
V_{0}(\lambda, v, \phi)=-\frac{1}{2} \lambda v^{2} \phi^{2}+\frac{\lambda}{4} \phi^{4}
$$

The one-loop Coleman-Weinberg correction $V_{\mathrm{CW}}$ is

$V_{\mathrm{CW}}(g, \lambda, v, \phi)=\sum_{b} n_{b} \frac{m_{b}^{4}(\phi)}{64 \pi^{2}}\left(\ln \frac{m_{b}^{2}(\phi)}{\mu_{R}^{2}}-C_{a}\right)$,

which we have written here in Landau gauge using the $\overline{\mathrm{MS}}$ renormalization scheme which gives $C_{a}=3 / 2(5 / 6)$ for scalars (gauge bosons). The sum on $b$ is over all bosons which have a $\phi$-dependent mass and $n_{b}$ is the total number of degrees of freedom of the boson. The final piece $V_{T \neq 0}$ is the finite temperature correction to the potential

$V_{T \neq 0}(g, \lambda, v, \phi, T)=\frac{T^{4}}{2 \pi^{2}} \sum_{b} n_{b} J_{b}\left(\frac{m_{b}^{2}(\phi)+\Pi_{b}(T)}{T^{2}}\right)$,

which includes a correction from resummed Daisy diagrams. The thermal function $J_{b}\left(x^{2}\right)$, the $\phi$-dependent masses $m_{b}(\phi)$, and the Debye masses $\Pi_{b}(T)$ are all given in the Supplemental Material [94]. As we will show later, in the $\mathrm{PS}^{3}$ model with $g \sim \mathcal{O}(1)$ and small $\lambda, V_{T \neq 0}$ naturally induces a thermal barrier which leads to a strong FOPT.

The subsequent SU(4) transitions at the scales $\Lambda_{\mathrm{II}}$ and $\Lambda_{\mathrm{IV}}$ are modeled by the more complicated breaking pattern $\mathrm{SU}(4) \times \mathrm{SU}(3)^{\prime} \rightarrow \mathrm{SU}(3)$, which is presented in the Supplemental Material [94].

Numerical procedure: The GW spectrum from a FOPT is described by four parameters [2,104-106]. These are the nucleation temperature $T_{n}$ which describes the onset of the phase transition, the strength $\alpha$, the inverse timescale $\beta$, and the bubble wall velocity $v_{w}$. While the full calculation of the bubble wall velocity is beyond the scope of this work, for illustration we simply assume that $v_{w} \sim 1$ [107].

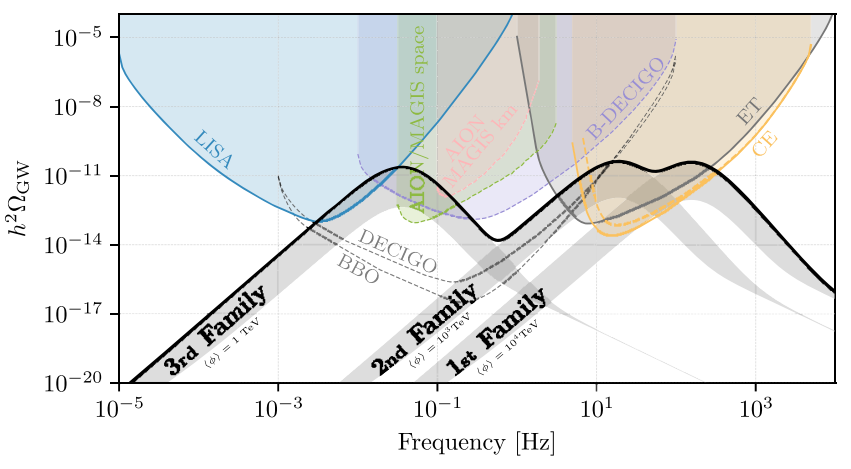

FIG. 2. Complete GW spectrum, which we term the "Triglav signature," following from three FOPTs in the PS $^{3}$ model. See the third section for details.

The remaining parameters we compute from the effective potential in Eq. (3) using the COSMOTRANSITIONS [108] package, the results of which we have confirmed using our own code based on the method of Ref. [109]. Thus, for a given set of model parameters $g, \lambda, v$ we compute the corresponding $\mathrm{GW}$ parameters $\alpha, \beta, T_{\mathrm{n}}$ which allows us to obtain the GW spectrum from a template function extracted from lattice simulation [110-112]. We then are able to perform a standard signal-to-noise ratio (SNR) analysis to determine the detectability of the signal, see, e.g.. Ref. [113]. More details can be found in the Supplemental Material [94].

Results: We show in Fig. 2 a benchmark multipeaked GW signal where the first two transitions would be detectable in ET/CE and the final $\mathrm{TeV}$ scale phase transition would be detectable in LISA. Remarkably, the predicted $\mathrm{PS}^{3}$ symmetry breaking scales (Fig. 1) correspond to peak frequencies in the optimal range for experiments. The solid black line is the total signal which is the combination of the individual spectra and corresponds to the nominal LISA recommendation for modeling GW formation and propagation [2]. The gray bands correspond to a conservative treatment of the sound wave contribution which illustrates the amount of theoretical uncertainty.

In the benchmark signal of Fig. 2, different peaks are obtained by appropriately varying the VEVs $\left(1,10^{3}, 10^{4} \mathrm{TeV}\right)$, as well as the effective relativistic degrees of freedom in the plasma. The renormalization group evolution of $\mathrm{PS}^{3}$ unambiguously determines the values of all gauge couplings at the relevant scales starting from the benchmark input $g_{4,3}\left(\Lambda_{\mathrm{IV}}\right)=2, g_{4,2}\left(\Lambda_{\mathrm{II}}\right)=\sqrt{2}$, and matching to the strong coupling at the scale $\Lambda_{\mathrm{IV}}$ [114]. The SU(4) coupling at the third site $g_{4,3}\left(\Lambda_{\mathrm{IV}}\right)$ is compatible with the current flavor anomalies as discussed in the Supplemental Material [94]. Finally, the three quartic couplings are set to $\lambda\left(\Lambda_{\mathrm{IV}}\right)=10^{-2}, \lambda\left(\Lambda_{\mathrm{II}}\right)=10^{-2}$, and $\lambda\left(\Lambda_{\mathrm{I}}\right)=0.5 \times 10^{-2}$.

To assess how generic GW signatures are in $\mathrm{PS}^{3}$, we show as an example in Fig. 3 the detectability of the GW 


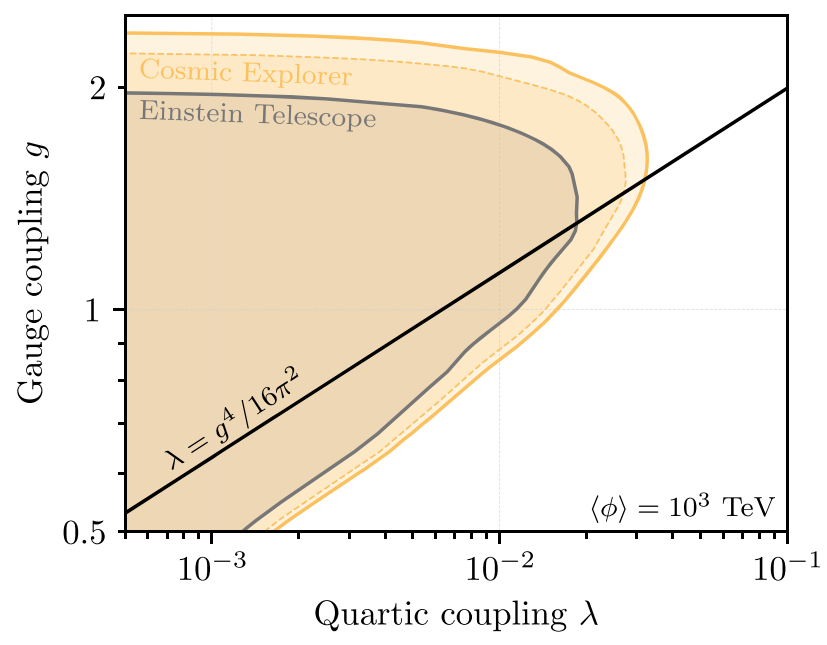

FIG. 3. Detectability of GW from a FOPT in the $4 \rightarrow 3$ simplified model. See the third section for details.

spectrum computed in ET and CE, as a function of $g$ and $\lambda$ for a fixed VEV of $10^{3} \mathrm{TeV}$. These regions were computed using our simplified $4 \rightarrow 3$ model to calculate the GW parameters and spectrum, after which a detectability analysis is performed where we require an SNR of 5 to obtain the boundaries. One can see immediately from Fig. 3 that significant parameter space exists which allows for a FOPT producing an observable GW signal without tuning [115]. Furthermore, the best GW signatures are given for (i) gauge couplings of $\mathcal{O}(1)$ and (ii) small quartic coupling.

Interestingly enough as discussed in the second section, both of these conditions are generic predictions of $\mathrm{PS}^{3}$ because (i) it embeds the strong gauge group and (ii) the natural size of the quartic is set by the one-loop ColemanWeinberg correction from the gauge sector. Indeed, the solid black line of Fig. 3 which falls nicely into the detectable region shows the expected size of $\lambda \approx$ $g^{4} / 16 \pi^{2}$ as would be generated from renormalization group flow. We have verified that $\mathrm{SU}(4) \times \mathrm{SU}(3)^{\prime} \rightarrow \mathrm{SU}(3)$ breaking pattern leads to qualitatively similar results.

Conclusions. - The peculiar pattern of hierarchical fermion masses which span many orders of magnitude is one of the longest standing puzzles in fundamental physics, the solution of which might require radical new approaches beyond colliders. In this Letter we propose for the first time that a multipeaked stochastic gravitational wave signature (where the ratios of peak frequencies follow the flavor hierarchies) could provide such a probe.

This idea is best illustrated in the context of the recently proposed PS $^{3}$ model for flavor hierarchies [63], motivated also in part by the current $B$-meson anomalies. Here, the successful quark-lepton unification of the original PatiSalam model is made compatible with flavor data by dimensional deconstruction onto three sites, one for each generation of SM fermions.
We show that the parameters of the $\mathrm{PS}^{3}$ model generically yield strongly first-order phase transitions as the gauge symmetry is sequentially broken down to the SM in hierarchical steps. Remarkably, the peak frequencies of the resulting GW spectra as determined by the VEVs fall precisely into the projected sensitivity range of future experiments. As we have argued, these are nearly inevitable predictions of the model as constructed. Such a spectacular signal, if observed, would offer a unique opportunity to probe the origins of the flavor hierarchies at energy scales which are currently inaccessible to colliders.

We thank Kfir Blum, Moritz Breitbach, Djuna Croon, Javier Fuentes-Martín, Gino Isidori, Andrey Katz, Joachim Kopp, Pedro Schwaller, and Marko Simonović for insightful discussions. T. O. has received funding from the European Research Council (ERC) under the European Union's Horizon 2020 research and innovation programme (Grant Agreement No. 637506, “ $\nu$ Directions") awarded to Joachim Kopp.

*admir.greljo@cern.ch

†toby.opferkuch@cern.ch

tbstefan@uni-mainz.de

[1] B. P. Abbott et al. (LIGO Scientific and Virgo Collaborations), Observation of Gravitational Waves from a Binary Black Hole Merger, Phys. Rev. Lett. 116, 061102 (2016).

[2] C. Caprini et al., Science with the space-based interferometer eLISA. II: Gravitational waves from cosmological phase transitions, J. Cosmol. Astropart. Phys. 04 (2016) 001.

[3] K. Yagi and N. Seto, Detector configuration of DECIGO/ $\mathrm{BBO}$ and identification of cosmological neutron-star binaries, Phys. Rev. D 83, 044011 (2011); Erratum, Phys. Rev. D 95, 109901 (2017).

[4] S. Kawamura et al., The Japanese space gravitational wave antenna: DECIGO, Classical Quantum Gravity 28, 094011 (2011).

[5] M. Punturo et al., The Einstein Telescope: A thirdgeneration gravitational wave observatory, Classical Quantum Gravity 27, 194002 (2010).

[6] B. Sathyaprakash et al., Scientific objectives of Einstein telescope, Classical Quantum Gravity 29, 124013 (2012); Erratum, Classical Quantum Gravity 30, 079501(E) (2013).

[7] B. P. Abbott et al. (LIGO Scientific Collaboration), Exploring the sensitivity of next generation gravitational wave detectors, Classical Quantum Gravity 34, 044001 (2017).

[8] P. W. Graham, J. M. Hogan, M. A. Kasevich, and S. Rajendran, Resonant mode for gravitational wave detectors based on atom interferometry, Phys. Rev. D 94, 104022 (2016).

[9] P. W. Graham, J. M. Hogan, M. A. Kasevich, S. Rajendran, and R.W. Romani (MAGIS Collaboration), Mid-band gravitational wave detection with precision atomic sensors, arXiv:1711.02225. 
[10] B. Allen, The stochastic gravity wave background in inflationary universe models, Phys. Rev. D 37, 2078 (1988).

[11] M. S. Turner and F. Wilczek, Relic Gravitational Waves and Extended Inflation, Phys. Rev. Lett. 65, 3080 (1990).

[12] M. Maggiore, Gravitational wave experiments and early universe cosmology, Phys. Rep. 331, 283 (2000).

[13] D. J. Weir, Gravitational waves from a first order electroweak phase transition: A brief review, Phil. Trans. R. Soc. A 376, 20170126 (2018).

[14] D. Curtin, P. Meade, and H. Ramani, Thermal resummation and phase transitions, Eur. Phys. J. C 78, 787 (2018).

[15] A. Katz and M. Perelstein, Higgs couplings and electroweak phase transition, J. High Energy Phys. 07 (2014) 108.

[16] M. J. Baker and J. Kopp, Dark Matter Decay Between Phase Transitions at the Weak Scale, Phys. Rev. Lett. 119, 061801 (2017).

[17] I. Baldes and G. Servant, High scale electroweak phase transition: Baryogenesis \& symmetry non-restoration, J. High Energy Phys. 10 (2018) 053.

[18] J. Ellis, M. Lewicki, and J.M. No, On the maximal strength of a first-order electroweak phase transition and its gravitational wave signal, J. Cosmol. Astropart. Phys. 04 (2019) 003.

[19] E. Madge and P. Schwaller, Leptophilic dark matter from gauged lepton number: Phenomenology and gravitational wave signatures, J. High Energy Phys. 02 (2019) 048.

[20] A. Beniwal, M. Lewicki, M. White, and A. G. Williams, Gravitational waves and electroweak baryogenesis in a global study of the extended scalar singlet model, J. High Energy Phys. 02 (2019) 183.

[21] D. Croon, T. E. Gonzalo, and G. White, Gravitational waves from a Pati-Salam phase transition, J. High Energy Phys. 02 (2019) 083.

[22] D. Croon, V. Sanz, and G. White, Model discrimination in gravitational wave spectra from dark phase transitions, J. High Energy Phys. 08 (2018) 203.

[23] V. Brdar, A.J. Helmboldt, and J. Kubo, Gravitational waves from first-order phase transitions: LIGO as a window to unexplored seesaw scales, J. Cosmol. Astropart. Phys. 02 (2019) 021.

[24] A. Addazi, A. Marcianò, and R. Pasechnik, Probing transelectroweak first order phase transitions from gravitational waves, MDPI Phys. 1, 92 (2019).

[25] M. Breitbach, J. Kopp, E. Madge, T. Opferkuch, and P. Schwaller, Dark, cold, and noisy: constraining secluded hidden sectors with gravitational waves, J. Cosmol. Astropart. Phys. 07 (2019) 007.

[26] A. Angelescu and P. Huang, Multistep strongly first order phase transitions from new fermions at the $\mathrm{TeV}$ scale, Phys. Rev. D 99, 055023 (2019).

[27] A. Alves, T. Ghosh, H.-K. Guo, K. Sinha, and D. Vagie, Collider and gravitational wave complementarity in exploring the singlet extension of the Standard Model, J. High Energy Phys. 04 (2019) 052.

[28] K. Kannike and M. Raidal, Phase transitions and gravitational wave tests of pseudo-goldstone dark matter in the softly broken U(1) scalar singlet model, Phys. Rev. D 99, 115010 (2019).
[29] M. Fairbairn, E. Hardy, and A. Wickens, Hearing without seeing: Gravitational waves from hot and cold hidden sectors, J. High Energy Phys. 07 (2019) 044.

[30] T. Hasegawa, N. Okada, and O. Seto, Gravitational waves from the minimal gauged $U(1)_{B-L}$ model, Phys. Rev. D 99, 095039 (2019).

[31] D. Dunsky, L. J. Hall, and K. Harigaya, Dark Matter, dark radiation and gravitational waves from mirror Higgs parity, J. High Energy Phys. 02 (2020) 078.

[32] P. Athron, C. Balazs, A. Fowlie, G. Pozzo, G. White, and Y. Zhang, Strong first-order phase transitions in the NMSSM-A comprehensive survey, J. High Energy Phys. 11 (2019) 151.

[33] S. De Curtis, L. Delle Rose, and G. Panico, Composite dynamics in the early Universe, J. High Energy Phys. 12 (2019) 149.

[34] A. Addazi, A. Marcianò, A. P. Morais, R. Pasechnik, R. Srivastava, and J. W. F. Valle, Gravitational footprints of massive neutrinos and lepton number breaking, arXiv: 1909.09740.

[35] T. Alanne, T. Hugle, M. Platscher, and K. Schmitz, A fresh look at the gravitational-wave signal from cosmological phase transitions, J. High Energy Phys. 03 (2020) 004.

[36] L. Bian, W. Cheng, H.-K. Guo, and Y. Zhang, Gravitational waves triggered by $B-L$ charged hidden scalar and leptogenesis, arXiv:1907.13589.

[37] A. Mohamadnejad, Gravitational waves from scaleinvariant vector dark matter model: Probing below the neutrino-floor, Eur. Phys. J. C 80, 197 (2020).

[38] N. Okada and O. Seto, Probing the seesaw scale with gravitational waves, Phys. Rev. D 98, 063532 (2018).

[39] T. Vachaspati and A. Vilenkin, Gravitational radiation from cosmic strings, Phys. Rev. D 31, 3052 (1985).

[40] T. Damour and A. Vilenkin, Gravitational radiation from cosmic (super)strings: Bursts, stochastic background, and observational windows, Phys. Rev. D 71, 063510 (2005).

[41] J. J. Blanco-Pillado, K. D. Olum, and B. Shlaer, The number of cosmic string loops, Phys. Rev. D 89, 023512 (2014).

[42] J. A. Dror, T. Hiramatsu, K. Kohri, H. Murayama, and G. White, Testing Seesaw and Leptogenesis with Gravitational Waves, Phys. Rev. Lett. 124, 041804 (2020).

[43] S. Y. Khlebnikov and I. I. Tkachev, Relic gravitational waves produced after preheating, Phys. Rev. D 56, 653 (1997).

[44] R. Easther and E. A. Lim, Stochastic gravitational wave production after inflation, J. Cosmol. Astropart. Phys. 04 (2006) 010.

[45] R. Easther, J. T. Giblin, Jr., and E. A. Lim, Gravitational Wave Production at the End of Inflation, Phys. Rev. Lett. 99, 221301 (2007).

[46] J. Garcia-Bellido and D. G. Figueroa, A Stochastic Background of Gravitational Waves from Hybrid Preheating, Phys. Rev. Lett. 98, 061302 (2007).

[47] J. Garcia-Bellido, D. G. Figueroa, and A. Sastre, A gravitational wave background from reheating after hybrid inflation, Phys. Rev. D 77, 043517 (2008).

[48] J.-F. Dufaux, G. Felder, L. Kofman, and O. Navros, Gravity waves from tachyonic preheating after hybrid inflation, J. Cosmol. Astropart. Phys. 03 (2009) 001. 
[49] C. S. Machado, W. Ratzinger, P. Schwaller, and B. A. Stefanek, Audible axions, J. High Energy Phys. 01 (2019) 053.

[50] M. Sasaki, T. Suyama, T. Tanaka, and S. Yokoyama, Primordial black holes-perspectives in gravitational wave astronomy, Classical Quantum Gravity 35, 063001 (2018).

[51] L. Barack et al., Black holes, gravitational waves and fundamental physics: A roadmap, Classical Quantum Gravity 36, 143001 (2019).

[52] N. Bartolo, V. De Luca, G. Franciolini, M. Peloso, D. Racco, and A. Riotto, Testing primordial black holes as dark matter with LISA, Phys. Rev. D 99, 103521 (2019).

[53] B. Grinstein, M. Redi, and G. Villadoro, Low scale flavor gauge symmetries, J. High Energy Phys. 11 (2010) 067.

[54] R. Alonso, M. B. Gavela, G. Isidori, and L. Maiani, Neutrino mixing and masses from a minimum principle, J. High Energy Phys. 11 (2013) 187.

[55] D. Guadagnoli, R. N. Mohapatra, and I. Sung, Gauged flavor group with left-right symmetry, J. High Energy Phys. 04 (2011) 093.

[56] R. Alonso, M. B. Gavela, L. Merlo, and S. Rigolin, On the scalar potential of minimal flavour violation, J. High Energy Phys. 07 (2011) 012.

[57] R. Alonso, M. B. Gavela, D. Hernandez, and L. Merlo, On the potential of leptonic minimal flavour violation, Phys. Lett. B 715, 194 (2012).

[58] E. Nardi, Naturally large Yukawa hierarchies, Phys. Rev. D 84, 036008 (2011).

[59] J. R. Espinosa, C. S. Fong, and E. Nardi, Yukawa hierarchies from spontaneous breaking of the $S U(3)_{L} \times$ $S U(3)_{R}$ flavour symmetry?, J. High Energy Phys. 02 (2013) 137.

[60] T. Feldmann, F. Hartmann, W. Kilian, and C. Luhn, Combining Pati-Salam and flavour symmetries, J. High Energy Phys. 10 (2015) 160.

[61] F. Bishara, A. Greljo, J. F. Kamenik, E. Stamou, and J. Zupan, Dark matter and gauged flavor symmetries, J. High Energy Phys. 12 (2015) 130.

[62] A. Crivellin, J. Fuentes-Martin, A. Greljo, and G. Isidori, Lepton flavor non-universality in B decays from dynamical Yukawas, Phys. Lett. B 766, 77 (2017).

[63] M. Bordone, C. Cornella, J. Fuentes-Martin, and G. Isidori, A three-site gauge model for flavor hierarchies and flavor anomalies, Phys. Lett. B 779, 317 (2018).

[64] J. P. Lees et al. (BABAR Collaboration), Measurement of an excess of $\bar{B} \rightarrow D^{(*)} \tau^{-} \bar{\nu}_{\tau}$ decays and implications for charged Higgs bosons, Phys. Rev. D 88, 072012 (2013).

[65] S. Hirose et al. (Belle Collaboration), Measurement of the $\tau$ Lepton Polarization and $R\left(D^{*}\right)$ in the Decay $\bar{B} \rightarrow D^{*} \tau^{-} \bar{\nu}_{\tau}$, Phys. Rev. Lett. 118, 211801 (2017).

[66] R. Aaij et al. (LHCb Collaboration), Measurement of the Ratio of Branching Fractions $\mathcal{B}\left(\bar{B}^{0} \rightarrow D^{*+} \tau^{-} \bar{\nu}_{\tau}\right) /$ $\mathcal{B}\left(\bar{B}^{0} \rightarrow D^{*+} \mu^{-} \bar{\nu}_{\mu}\right)$, Phys. Rev. Lett. 115, 111803 (2015).

[67] R. Aaij et al. (LHCb Collaboration), Test of Lepton Universality Using $\mathrm{B}^{+} \rightarrow \mathrm{K}^{+} \ell^{+} \ell^{-}$Decays, Phys. Rev. Lett. 113, 151601 (2014).

[68] R. Aaij et al. (LHCb Collaboration), Test of lepton universality with $B^{0} \rightarrow K^{* 0} \ell^{+} \ell^{-}$decays, J. High Energy Phys. 08 (2017) 055.
[69] R. Aaij et al. (LHCb Collaboration), Measurement of Form-Factor-Independent Observables in the Decay $B^{0} \rightarrow K^{* 0} \mu^{+} \mu^{-}$, Phys. Rev. Lett. 111, 191801 (2013).

[70] R. Aaij et al. (LHCb Collaboration), Angular analysis of the $B^{0} \rightarrow K^{* 0} \mu^{+} \mu^{-}$decay using $3 \mathrm{fb}^{-1}$ of integrated luminosity, J. High Energy Phys. 02 (2016) 104.

[71] R. Aaij et al. (LHCb Collaboration), Search for LeptonUniversality Violation in $B^{+} \rightarrow K^{+} \ell^{+} \ell^{-}$Decays, Phys. Rev. Lett. 122, 191801 (2019).

[72] D. Buttazzo, A. Greljo, G. Isidori, and D. Marzocca, B-physics anomalies: A guide to combined explanations, J. High Energy Phys. 11 (2017) 044.

[73] This is arguably the most compelling UV picture that offers a coherent explanation of the current flavor anomalies. See, e.g., Refs. [74-79].

[74] A. Greljo and B. A. Stefanek, Third family quark-lepton unification at the TeV scale, Phys. Lett. B 782, 131 (2018).

[75] L. Di Luzio, A. Greljo, and M. Nardecchia, Gauge leptoquark as the origin of B-physics anomalies, Phys. Rev. D 96, 115011 (2017).

[76] M. Bordone, C. Cornella, J. Fuentes-Martín, and G. Isidori, Low-energy signatures of the $\mathrm{PS}^{3}$ model: From $B$-physics anomalies to LFV, J. High Energy Phys. 10 (2018) 148.

[77] C. Cornella, J. Fuentes-Martin, and G. Isidori, Revisiting the vector leptoquark explanation of the B-physics anomalies, J. High Energy Phys. 07 (2019) 168.

[78] M. Blanke and A. Crivellin, $B$ Meson Anomalies in a PatiSalam Model within the Randall-Sundrum Background, Phys. Rev. Lett. 121, 011801 (2018).

[79] J. Fuentes-Martín, M. Reig, and A. Vicente, Strong CP problem with low-energy emergent QCD: The 4321 case, Phys. Rev. D 100, 115028 (2019).

[80] J. C. Pati and A. Salam, Lepton number as the fourth color, Phys. Rev. D 10, 275 (1974); Erratum, Phys. Rev. D 11, 703(E) (1975).

[81] N. Arkani-Hamed, A. G. Cohen, and H. Georgi, Electroweak symmetry breaking from dimensional deconstruction, Phys. Lett. B 513, 232 (2001).

[82] We propose a slight variation of the original model breaking $\left[\mathrm{SU}(2)_{R}\right]_{1}$ before inflation effectively solving the monopole problem of low-scale PS models [83]. $\mathrm{PS}_{1}^{\prime}$ in Fig. 1 is defined as $\left[\mathrm{SU}(4) \times \mathrm{SU}(2)_{L} \times \mathrm{U}(1)\right]_{1}$.

[83] R. Jeannerot, S. Khalil, G. Lazarides, and Q. Shafi, Inflation and monopoles in supersymmetric $\mathrm{SU}(4) \mathrm{C} \times$ $\mathrm{SU}(2)(\mathrm{L}) \times \mathrm{SU}(2)(\mathrm{R})$, J. High Energy Phys. 10 (2000) 012.

[84] R. Barbieri, G. Isidori, J. Jones-Perez, P. Lodone, and D. M. Straub, $U(2)$ and minimal flavour violation in supersymmetry, Eur. Phys. J. C 71, 1725 (2011).

[85] G. Valencia and S. Willenbrock, Quark-lepton unification and rare meson decays, Phys. Rev. D 50, 6843 (1994).

[86] A. D. Smirnov, Mass limits for scalar and gauge leptoquarks from $K_{L}^{0} \rightarrow e^{\mp} \mu^{ \pm}, B^{0} \rightarrow e^{\mp} \tau^{ \pm}$-decays, Mod. Phys. Lett. A 22, 2353 (2007).

[87] A. V. Kuznetsov, N. V. Mikheev, and A. V. Serghienko, The third type of fermion mixing in the lepton and quark interactions with leptoquarks, Int. J. Mod. Phys. A 27, 1250062 (2012). 
[88] G. F. Giudice, G. Isidori, A. Salvio, and A. Strumia, Softened gravity and the extension of the standard model up to infinite energy, J. High Energy Phys. 02 (2015) 137.

[89] A. D. Smirnov, Vector leptoquark mass limits and branching ratios of $K_{L}^{0}, B^{0}, B_{s} \rightarrow l_{i}^{+} l_{j}^{-}$decays with account of fermion mixing in leptoquark currents, Mod. Phys. Lett. A 33, 1850019 (2018).

[90] Another independent argument to keep the first two SSBs close to the bounds implied by FCNC is to avoid large tuning of the Higgs mass which is only partially screened from the first two sites.

[91] The SU(2) breakings at the scale $\Lambda_{\text {III }}$ may also produce stochastic GW signatures, but lie in a suboptimal frequency range for LISA and ET. This provides additional motivation for proposed intermediate frequency experiments such as atom interferometers or DECIGO.

[92] W. D. Goldberger and M. B. Wise, Modulus Stabilization with Bulk Fields, Phys. Rev. Lett. 83, 4922 (1999).

[93] N. Arkani-Hamed, H.-C. Cheng, P. Creminelli, and L. Randall, Extra Natural Inflation, Phys. Rev. Lett. 90, 221302 (2003).

[94] See the Supplemental Material at http://link.aps.org/ supplemental/10.1103/PhysRevLett.124.171802 for the higher-dimensional operators generating Yukawa interactions in Eq. (1) give a negligible correction to the effective potential. Regardless, we find that including fermions only increases the PT strength. The references found only in the Supplemental Material are [75-77,95103].

[95] M. Laine and A. Vuorinen, Basics of thermal field theory, Lect. Notes Phys. 925, 1 (2016).

[96] D. Comelli and J. R. Espinosa, Bosonic thermal masses in supersymmetry, Phys. Rev. D 55, 6253 (1997).

[97] M. González-Alonso, J. Martin Camalich, and K. Mimouni, Renormalization-group evolution of new physics contributions to (semi)leptonic meson decays, Phys. Lett. B 772, 777 (2017).

[98] F. Feruglio, P. Paradisi, and O. Sumensari, Implications of scalar and tensor explanations of $R_{D^{(*)}}$, J. High Energy Phys. 11 (2018) 191.

[99] Y.S. Amhis et al. (HFLAV Collaboration), Averages of $b$-hadron, $c$-hadron, and $\tau$-lepton properties as of 2018 , arXiv:1909.12524.

[100] A. M. Sirunyan et al. (CMS Collaboration), Search for heavy neutrinos and third-generation leptoquarks in hadronic states of two $\tau$ leptons and two jets in proton-proton collisions at $\sqrt{s}=13 \mathrm{TeV}$, J. High Energy Phys. 03 (2019) 170.

[101] C. Marzo, L. Marzola, and V. Vaskonen, Phase transition and vacuum stability in the classically conformal B-L model, Eur. Phys. J. C 79, 601 (2019).
[102] J. Ellis, M. Lewicki, J. M. No, and V. Vaskonen, Gravitational wave energy budget in strongly supercooled phase transitions, J. Cosmol. Astropart. Phys. 06 (2019) 024.

[103] M. Hindmarsh, S. J. Huber, K. Rummukainen, and D. J. Weir, Shape of the acoustic gravitational wave power spectrum from a first order phase transition, Phys. Rev. D 96, 103520 (2017).

[104] M. S. Turner, E. J. Weinberg, and L. M. Widrow, Bubble nucleation in first order inflation and other cosmological phase transitions, Phys. Rev. D 46, 2384 (1992).

[105] M. Kamionkowski, A. Kosowsky, and M. S. Turner, Gravitational radiation from first order phase transitions, Phys. Rev. D 49, 2837 (1994).

[106] C. Grojean and G. Servant, Gravitational waves from phase transitions at the electroweak scale and beyond, Phys. Rev. D 75, 043507 (2007).

[107] However, we have studied the impact of a reduced bubble wall velocity, e.g., $v_{\mathrm{w}} \sim 0.4$ as motivated by studies of the EWPT with a light Higgs. The dominant effect is a linear shift in peak frequency which can be compensated by a similar order-one shift in the VEV.

[108] C. L. Wainwright, CosmoTransitions: Computing cosmological phase transition temperatures and bubble profiles with multiple fields, Comput. Phys. Commun. 183, 2006 (2012).

[109] J. R. Espinosa, A fresh look at the calculation of tunneling actions, J. Cosmol. Astropart. Phys. 07 (2018) 036.

[110] S. J. Huber and T. Konstandin, Gravitational wave production by collisions: More bubbles, J. Cosmol. Astropart. Phys. 09 (2008) 022.

[111] M. Hindmarsh, S. J. Huber, K. Rummukainen, and D. J. Weir, Numerical simulations of acoustically generated gravitational waves at a first order phase transition, Phys. Rev. D 92, 123009 (2015).

[112] C. Caprini, R. Durrer, and G. Servant, The stochastic gravitational wave background from turbulence and magnetic fields generated by a first-order phase transition, J. Cosmol. Astropart. Phys. 12 (2009) 024.

[113] E. Thrane and J.D. Romano, Sensitivity curves for searches for gravitational-wave backgrounds, Phys. Rev. D 88, 124032 (2013).

[114] The breaking of $\mathrm{SU}(4)_{i} \times \mathrm{SU}(3)_{j} \rightarrow \mathrm{SU}(3)$ implies the matching condition $g_{3}^{-2}=g_{4, i}^{-2}+g_{3, j}^{-2}$.

[115] Larger gauge coupling leads to an effective potential that deforms faster as a function of temperature. This has two effects (i) larger $T_{n}$, leading to an increased radiation energy density and hence a suppression of $\alpha$ and (ii) an increased $\beta / H$. These effects decrease the amplitude and increase the peak frequency of the GW signal leading to the upper boundary of Fig. 3. 